\title{
Optimizing WiMAX: A Dynamic Strategy for Reallocation of Underutilized Downlink Sub-Frame to Uplink in TDD Mode
}

\author{
Abdul Qadir ANSARI ${ }^{1}$, Abdul Qadeer K. RAJPUT ${ }^{2}$, Adnan Ashraf ARAIN ${ }^{2}$, Manzoor HASHMANI ${ }^{2}$ \\ ${ }^{1}$ Wireless Core Network, Pakistan Telecommunication Company Limited, Pakistan \\ ${ }^{2}$ CREST-Research Group, IICT, Mehran University of Engineering and Technology, Jamshoro, Pakistan \\ E-mail:qadir.ansari@ptcl.net.pk,aqkrajput@muet.edu.pk,adnanlooking@ieee.org,mhashmani@yahoo.com \\ Received August 20, 2009; revised September 22, 2009; accepted November 1, 2009
}

\begin{abstract}
WiMAX networks experience sporadic congestion on uplink when applications running at subscriber stations need more bandwidth to transmit than allocated. With the fast proliferation of mobile Internet, the wireless community has been looking for a framework that can address the issue of impediment on uplink. Due to asymmetric behavior of Internet applications downlink sub-frame is expected to have longer duration as compared to uplink. According to IEEE 806.16 standard for WiMAX the segmentation of TDD frame between uplink and downlink can be dynamically redefined even at runtime. Research contributions so far lack in addressing an optimal strategy for readjustment of uplink and downlink sub-frame boundaries; based on traffic statistics. In this paper, we introduce a mechanism that allows uplink sub-frame to grow, borrowing resources from the downlink sub-frame, if the uplink utilization is high and the downlink is being underutilized. We present here, a framework to dynamically demarcate the TDD frame-duration between uplink and downlink. Proposed algorithm takes into account the present utilization of downlink and reallocates a certain quantum of free resources to uplink. This occurs when uplink observes shortage of bandwidth to transmit. We simulate some test scenarios using OPNET Modeler with and without dynamic reallocation capability. The results of our simulation confirm the effectiveness of proposed algorithm which observes a remarkable decrease in end-to-end packet delay. Also, we observe an improvement in throughput at uplink such that, the performance of downlink remains unaffected.
\end{abstract}

Keywords: WiMAX, Time Division Duplex (TDD), Quality of Service (QoS), End-to-End Packet Delay, Network Throughput

\section{Introduction}

The IEEE 802.16 family of standards specifies the air interface of fixed and mobile broadband wireless access (BWA) systems that support multimedia services. The IEEE 802.16-2004 standard, which was previously called $802.16 \mathrm{~d}$ or $802.16-\mathrm{REVd}$, was published for fixed access in October 2004. Good reviews of the standard can be found in [1-3]. The standard has been updated and extended to the $802.16 \mathrm{e}$ standard for mobile access, Mobile WiMAX, as of October 2005 [4]. Mobile WiMAX is a broadband wireless solution that enables convergence of mobile and fixed broadband networks. Mobile WiMAX technology is designed to be able to scale to work in different channelizations from 1.25 to $20 \mathrm{MHz}$ to comply with varied requirements.

The fundamental premise of the IEEE 802.16e MAC architecture is QoS on the move. With fast air interface, asymmetric downlink/uplink configuration capability, fine resource granularity and a flexible resource allocation mechanism, Mobile WiMAX can meet QoS requirements for a wide range of data services and applications [5].

IEEE 802.16e standard includes QoS support framework; however, it left undefined the details to ensure QoS guarantees, scheduling algorithms, uplink (UL) and downlink (DL) sub-frame allocation; for vendors as a motivation to device effective scheduling and resource allocation mechanisms to deliver QoS guarantees, especially for the real-time traffic. 
WiMAX networks support two types of duplexing modes to separate UL and DL communication; i.e. Time Division Duplex (TDD) and Frequency Division Duplex (FDD). In this paper we have focused on TDD mode where both UL and DL share same frequency and to separate downlink and uplink, time division multiple access (TDMA) is used.

The duration of DL and UL sub-frames may be decided once based on average traffic statistic expectations. However, it is also possible to tune the network configuration through real-time monitoring, and may readjust the uplink and downlink boundaries.

According to the standard, this segmentation can be dynamically adjusted even at runtime. Unfortunately, research contributions so far lacks in addressing an optimal strategy towards readjustment of UL and DL boundaries dynamically; while keeping the current traffic statistics in account. It is important to remember that asymmetric behavior of Internet applications intuitively ask for more duration of DL sub-frame as compared to UL. Also DL traffic behaviors could be controlled at serving Base Station (BS); but it is not true for UL.

We have introduced a mechanism in order to allow the UL sub-frame to "grow", borrowing resources from the DL sub-frame, if the UL utilization is high and the DL utilization is low. Our strategy is to keep the performance graph of downlink traffic unaffected by monitoring the downlink utilization and requirement. Moreover resources borrowed from DL will be relinquished as and when required at DL. The mechanism is tested in a controlled environment for it effectiveness. Simulation results confirmed the positive impact of this new capability on throughput and packet end-to-end delay on UL.

\section{WiMAX-Time Division Duplex}

The IEEE 802.16e-2005 supports both time division duplexing (TDD) and frequency division duplexing (FDD) modes. In TDD mode, the uplink and downlink transmission share the same frequency but do not transmit simultaneously. The frame, in Figure 1, is flexibly divided into a downlink sub-frame and an uplink subframe. The downlink sub-frame used to transmit data from a BS to SS. The uplink sub-frame carries SS traffic to the BS. The sub-frame is divided into mini slots, which is the minimum unit of data transfer in this level. Frames are broadcasted and during the downlink subframe, the SS picks up the data addressed to it. Media Access Protocol (MAP) messages are broadcasted at the beginning of each downlink sub-frame. There are two types of MAP messages, DL-MAP and UL-MAP. The DL-MAP described the usage of the downlink sub-frame whereas the UP-MAP tells which mini slot and how many mini slots are allocated to the specified SS for its trans- mission during the uplink sub-frame.

Mobile WiMAX profiles only consider the TDD mode of operation for the following reasons:

1) It allows dynamic reallocation of DL and UL radio resources to effectively support asymmetric traffic pattern that is common in Internet applications.

2) The allocation of radio resources in DL and UL is determined by the DL/UL switching point(s).

3) Both DL and UL are in the same frequency channel to yield better channel reciprocity and to better support link adaptation.

4) A single frequency channel in DL and UL can provide more flexibility for spectrum allocation.

As shown in Figure 2, the connection between a SS and $\mathrm{BS}$ is identified by a unique connection identifier (CID). One CID can correspond to an individual application or a number of applicants bundled together such as a group of users in the same building. CID also specifies polling schemes provided to the connection by the BS, which will result in QoS for the connection who owns this CID.

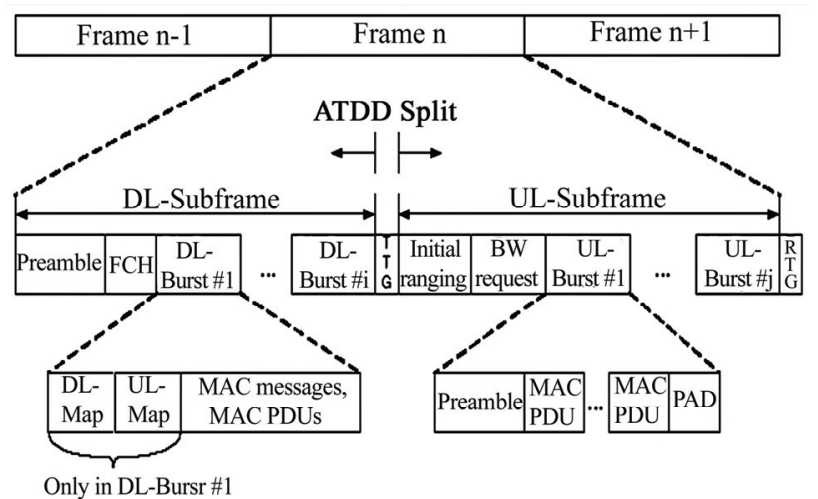

Figure 1. WIMAX TDD frame.

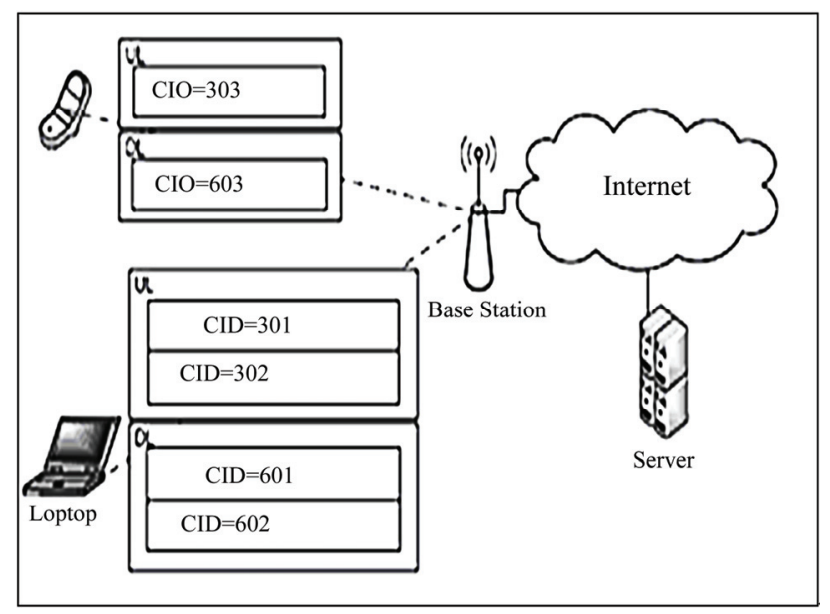

Figure 2. Connection (CID)-based WiMAX MAC layer [6]. 


\section{Quality of Service Support in WiMAX}

WiMAX with provisioned quality of service (QoS) for digital multimedia applications to mobile end users over wide area networks is the new frontier of telecommunications industry.

Before providing a certain type of a service, the base station and user-terminal first establish a unidirectional logical link between the peer MACs called a connection. The outbound MAC then associates packets traversing the MAC interface into a service flow to be delivered over the connection. The QoS parameters associated with the service flow define the transmission ordering and scheduling on the air interface. The connection-oriented QoS therefore, can provide accurate control over the air interface. Since the air interface is usually the bottleneck, the connection-oriented QoS can effectively enable the end-to-end QoS control. The service flow based QoS mechanism applies to both DL and UL to provide improved QoS in both directions.

The service flow parameters can be dynamically managed through MAC messages to accommodate the dynamic service demand. IEEE 802.16 MAC is connection-oriented. BS controls the access to the medium, bandwidth is granted to SS on demand. At the beginning of each frame, the BS schedules the uplink and downlink grants to meet the negotiated QoS requirements. Each SS learns the boundaries of its allocation under current uplink sub-frame via the UL-MAP message. The DL-MAP delivers the timetable of downlink grants in the downlink sub-frame [7].

\section{Related Research Review}

During last couple of years, many proposals for QoS service support in WiMAX networks were published [8-14]. Most of them are the solutions on the bandwidth allocation (with preset allocation of UL and DL subframe size [13]), flow scheduling and Adaptive Modulation and Coding Schemas [14] to optimize the performance of WiMAX network. In [15] a three-tier QoS framework is introduced where a Pre-scale Dynamic Resource Reservation (PDRR) is proposed to allocate frame bandwidth to UL sub-frame and DL sub-frame with pre-scaled bounds. In [16] the presented baseline network model is examined for fixed and dynamic reallocation, but under dynamic reallocation the resources are relinquished completely from UL and allocated back to DL when a certain preset threshold for DL occupancy is met. We have modified the algorithm and proposed a step-by-step allocation and similarly a step-by-step deallocation of UL resources back when certain threshold of DL occupancy is met.

\section{Proposed Strategy to Enable Reallocation Capability}

We provide here a basic mechanism that allows uplink sub-frame to grow, borrowing resources from the DL sub-frame, if the uplink utilization is high and the downlink is underutilized. Our strategy ensures that resources borrowed should be relinquished as and when required by DL in order to ensure that with this introduced capability the DL performance should not be affected. Our strategy is to observe the utilization of DL resources and if DL is underutilized and UL is starving for bandwidth; DL sub-frame duration may be reduced and UL subframe duration may be increased by a certain quantum of time.

\subsection{The Proposed Algorithm}

1) Baseline network scenario is simulated using OPNET Modeler 14.5 (Wireless Suite) to test the impact of new capability to redefine the boundaries of UL and DL sub-frames.

2) Observe the frame allocation information and the traffic behavior prior to the addition of the new capability. For example; a) Observe sub-frame utilization, and b) Application Performance in terms of throughput and end-to-end packet delay on UL

3) Implement a mechanism to change the uplink and downlink partition dynamically.

4) Implement an algorithm that performs re-allocation of the sub-frames.

5) Ensure the performance of DL traffic should remain unaffected with introduction of new capability.

6) Observe and analyze the results with the new capability.

7) Compare, analyze and summarize the simulation results with the baseline network results.

\subsection{Flowchart of our Proposed Algorithm}

Here, we set certain thresholds for UL and DL sub-frame utilization to decide whether or not the sub-frame duration need to be dynamically adjusted. The same is shown here, in the flowchart of our proposed algorithm in Figure 3.

The above flow chart shows how the evaluation of the sub-frames utilization is used to determine whether increase the UL sub-frame size or prohibit any readjustment of sub-frame duration.

\subsection{Pseudo Code of Proposed Algorithm}

1) Check DL Sub-Frame Utilization.

a) Is DL already reduced 


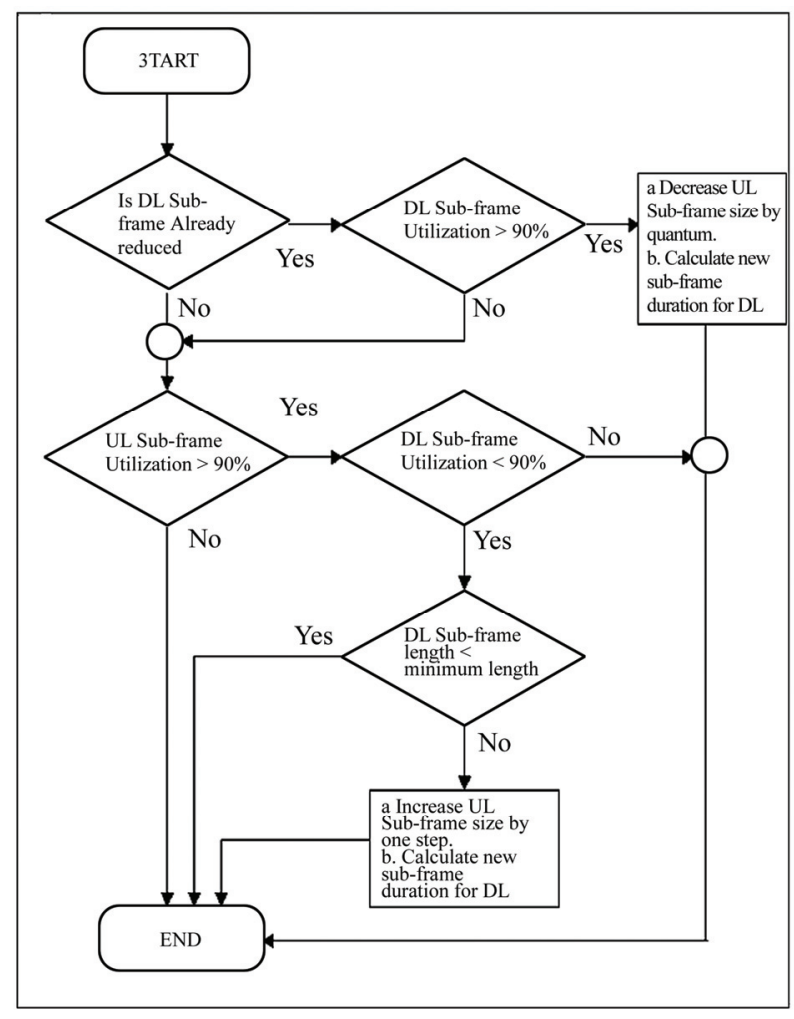

Figure 3. Proposed scheme- flow chart.

b) Is DL utilization is below threshold

c) If DL is already reduced but utilization is still under threshold go to Step 2.

d) Else revert back last reduction in DL

e) Reset the UL and DL sub-fame boundaries to the previous values for each respectively and go to Step 4 .

2) Check UL Sub-Frame Utilization.

a) Is UL sub-frame utilization above threshold?

b) Is DL under utilized?

c) If both conditions $a$ and $b$ are TRUE then check condition in $\mathrm{d}$.

d) Check DL sub-frame length $<$ minimum allowable length

e) If condition fails in $d$ then go to Step 4 .

f) Else go to Step 3 .

3) Increase UL Frame Size by One Step.

a. Update UL and DL sub-fame boundaries

4) EXIT.

\section{Our Proposed Setup for Simulation}

The baseline network is composed of one WiMAX cell with four SS nodes. All SS nodes have an uplink application load of $250 \mathrm{Kbps}$ for a total of $1 \mathrm{Mbps}$. At specific times, the Server generates $600 \mathrm{Kbps}$ of application traffic directed to SS-0 and SS-1; this creates a total downlink application load of $1.2 \mathrm{Mbps}$. The cell uses Scalable OFDMA frame with 512 sub-carriers and of 5 milliseconds duration. Uplink sub-frame is set to 12 symbol times (i.e. frame columns). Downlink sub-frame is assigned 34 symbol times. For QPSK 1/2, the capacity expected is Uplink: $\sim 0.6 \mathrm{Mbps}$. Downlink: 2.5 Mbps.

\subsection{Preset Sub-frame Allocation between UL and DL}

\subsubsection{Traffic Behavior}

Following graph (Figure 4) shows MAC load and throughput for DL. It can be easily observed that DL MAC load and throughput is same i.e. 1.2 Mbps. Total DL capacity is $2.5 \mathrm{Mbps}$; thus there is enough capacity at DL to successfully transport the DL load.

Moreover the ETE packet delay remains between 6 10 milliseconds which is fairly under acceptable range, as shown in Figure 5.

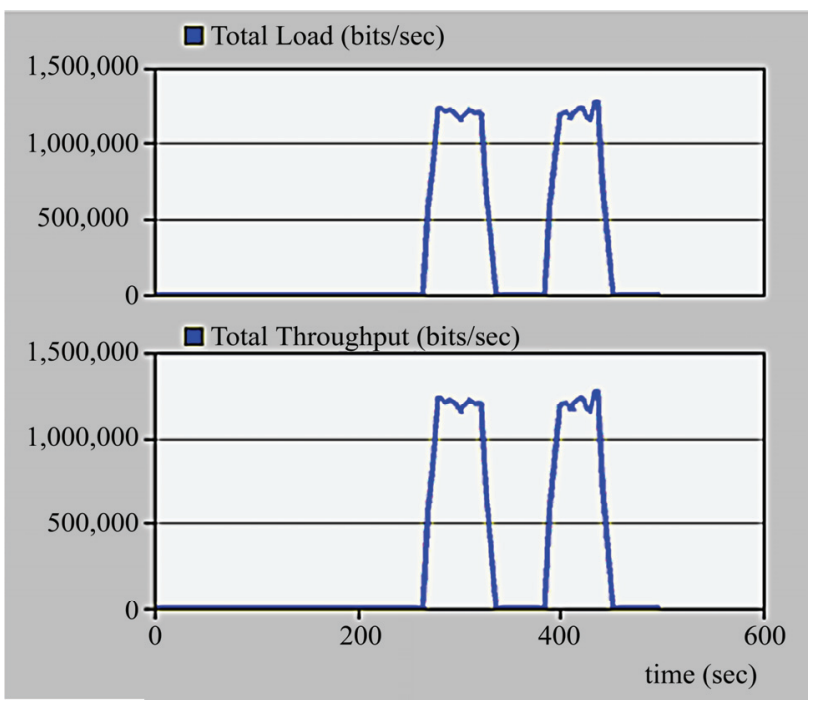

Figure 4. DL traffic load and throughput.

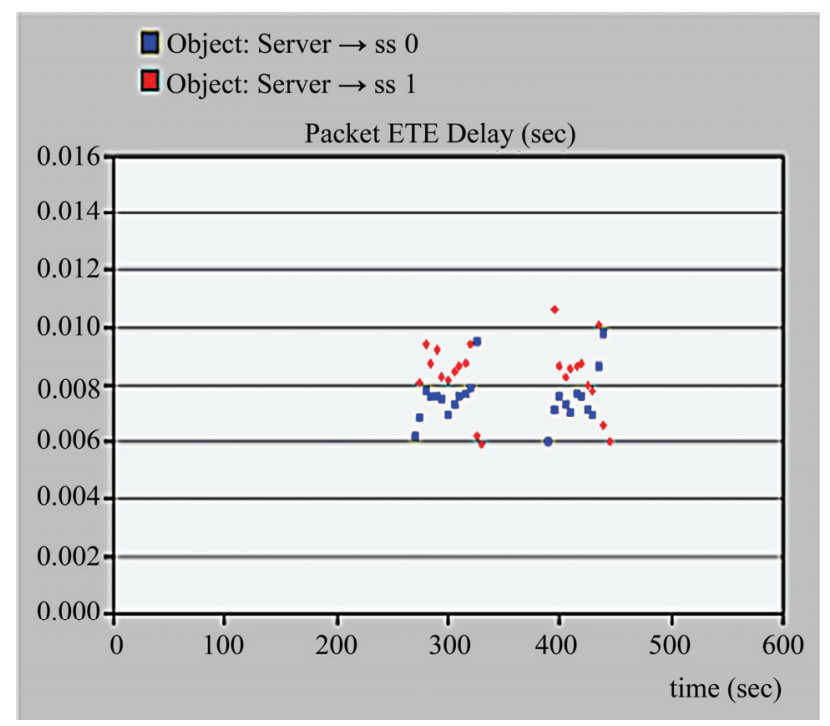

Figure 5. End-to-end packet delay (DL). 


\subsubsection{UL Traffic Behavior}

Following graph (in Figure 6) shows MAC load and throughput for UL. We observe that UL MAC load is $\sim 1$ Mbps and throughput is $0.56 \mathrm{Mbps}$. UL capacity is 0.6 Mbps; thus the offered load is exceeding the capacity; which results in high application delays ranging from 3.5 to 3.75 seconds (Figure 7). Thus UL is running out of resources and do not have enough capacity to accommodate any further load.

\subsubsection{Statistics of Usage and Usable Sizes (UL and DL)}

Statistics results are also collected, in Figure 8, for data burst percentage utilization and sub-frame usable size for UL and DL.

From above diagram it is evident that DL usage is about $60 \%$; however UL usage is $100 \%$. Also usable

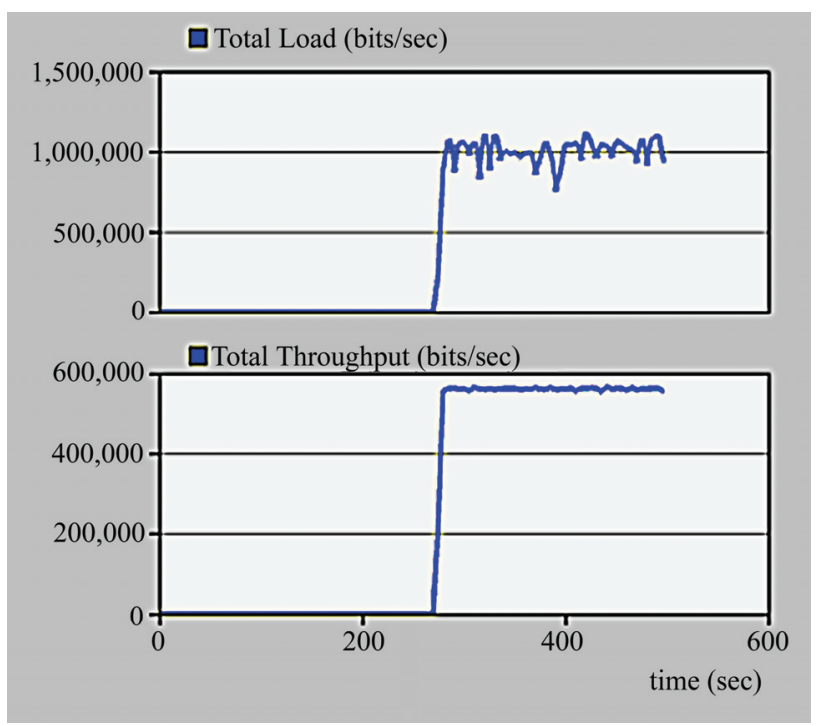

Figure 6. UL traffic load and throughput.

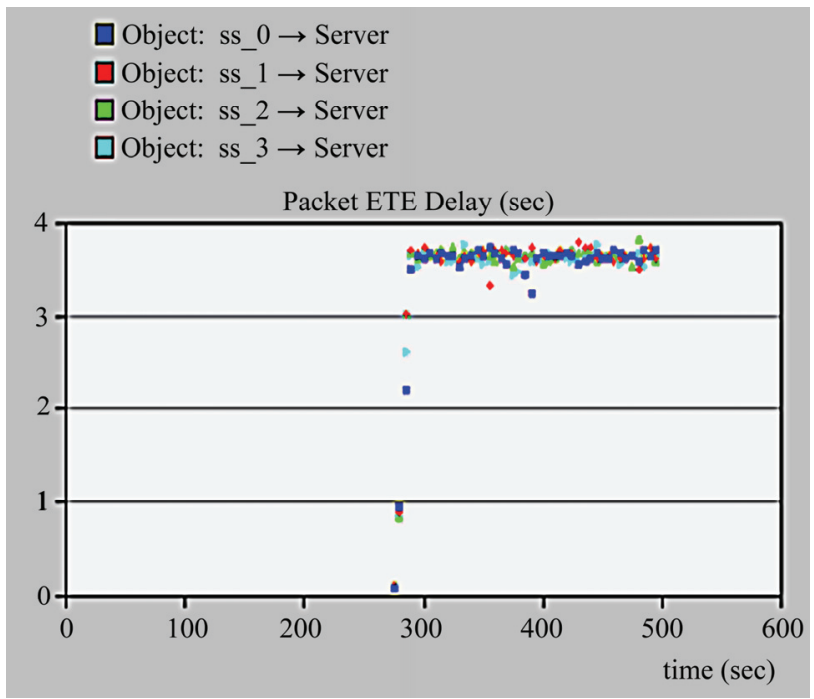

Figure 7. End-to-end packet delay (UL).

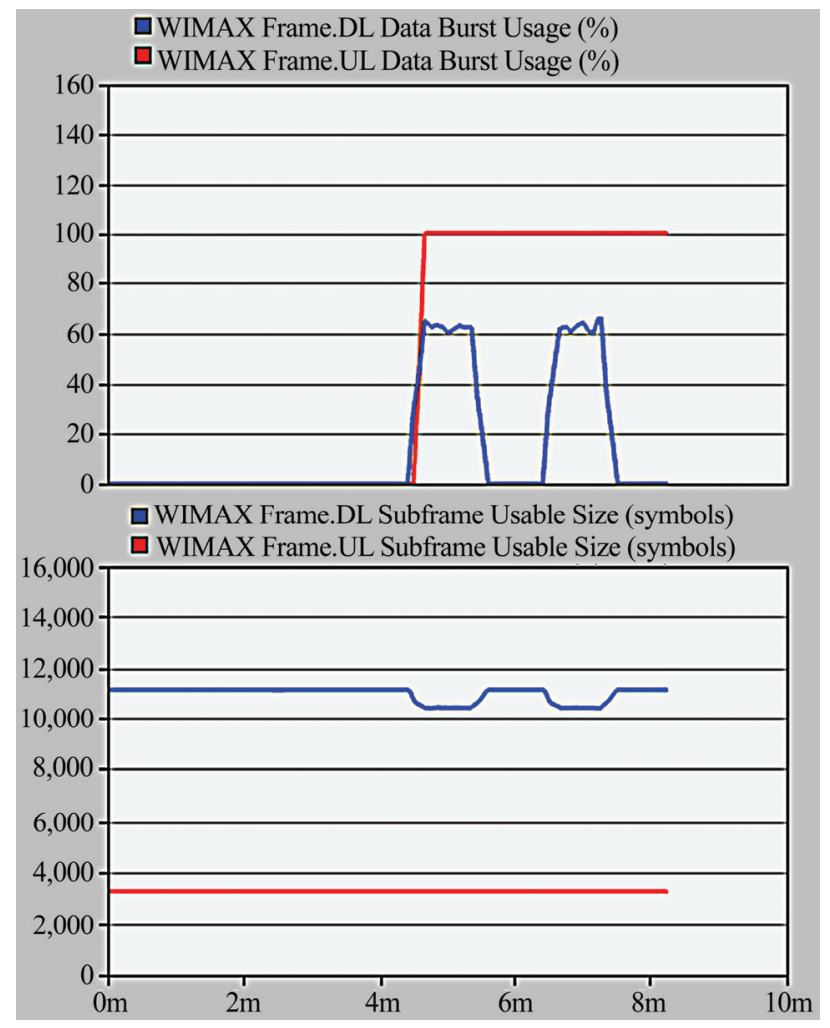

Figure 8. Data burst usage and sub-frame usable size.

sub-frame size for UL is merely $\sim 3.3 \mathrm{~K}$ symbols and same for DL is nearly $11.3 \mathrm{~K}$ Symbols.

\subsection{Dynamic Sub-Frame Allocation between UL and DL}

\subsubsection{Traffic Behavior}

The performance of DL traffic remained unaffected as desired; i.e. 1.2 Mbps MAC load and throughput (Figure 9). Comparison could also be found in second frame. Application end-to-end packet delay is observed to remain under $\sim 8$ milliseconds (Figure 10 ).

\subsubsection{UL Traffic Behavior}

Here we can confirm the major improvement in throughput at UL. Comparison is presented between constant and adaptive schemas. It is evident that load and throughput are almost aligned on UL as well (Figure 11). Now UL transports almost all the traffic originated form $\mathrm{MS}$ and directed to BS.

This improvement has also resulted in remarkable decrease in packet end-to-end delay on UL; which has now reduced to $\sim 7$ milliseconds from 3 4 seconds (Figure 12).

\subsubsection{Statistics of Usage and Usable Sizes (UL and DL)}

From UL and DL data burst usage and Usable size (Fig- 


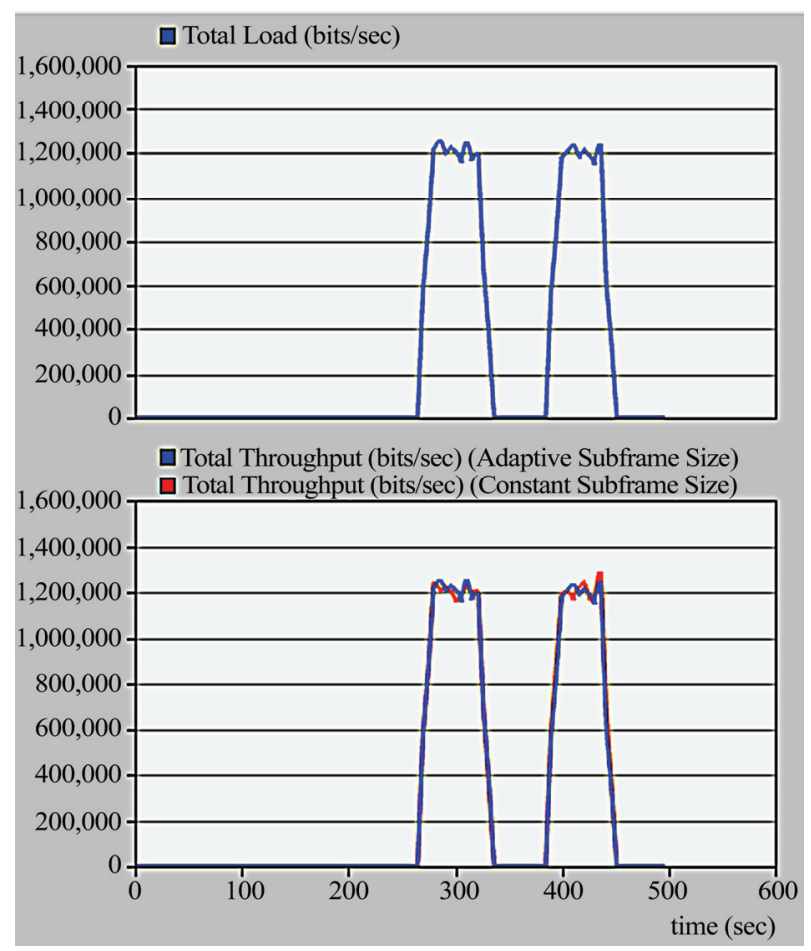

Figure 9. DL traffic load and throughput.

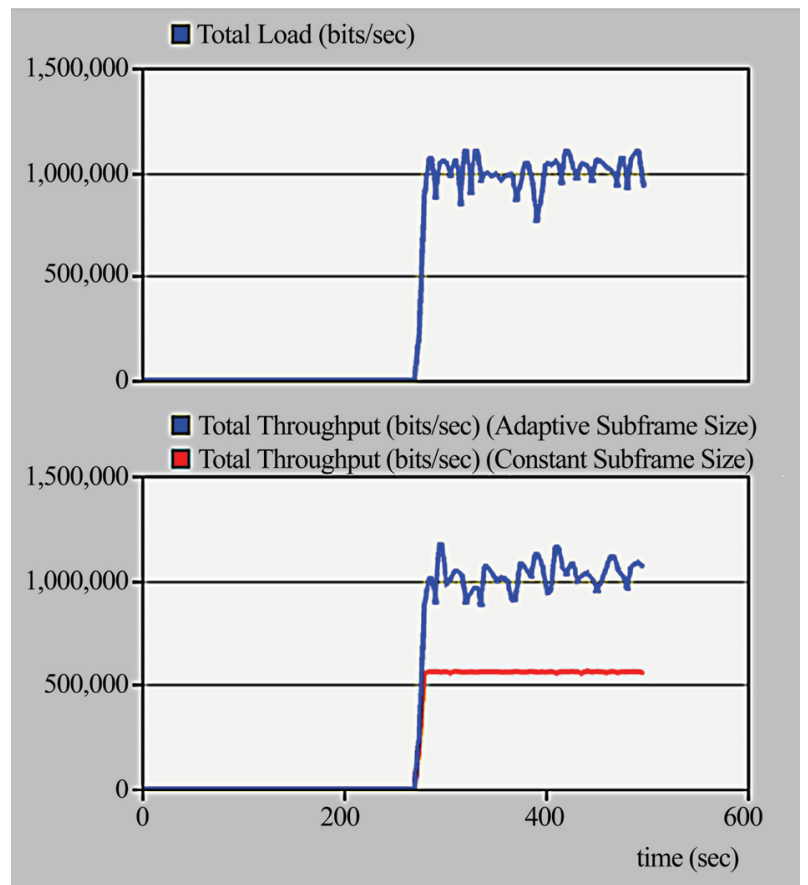

Figure 11. UL traffic load and throughput.

ure 13) statistics it is evident that our scheme worked well and effectively utilized the free DL bandwidth at UL. With our strategy UL usage has improved because the UL usable capacity increases to maximum when DL utilization is lowest.

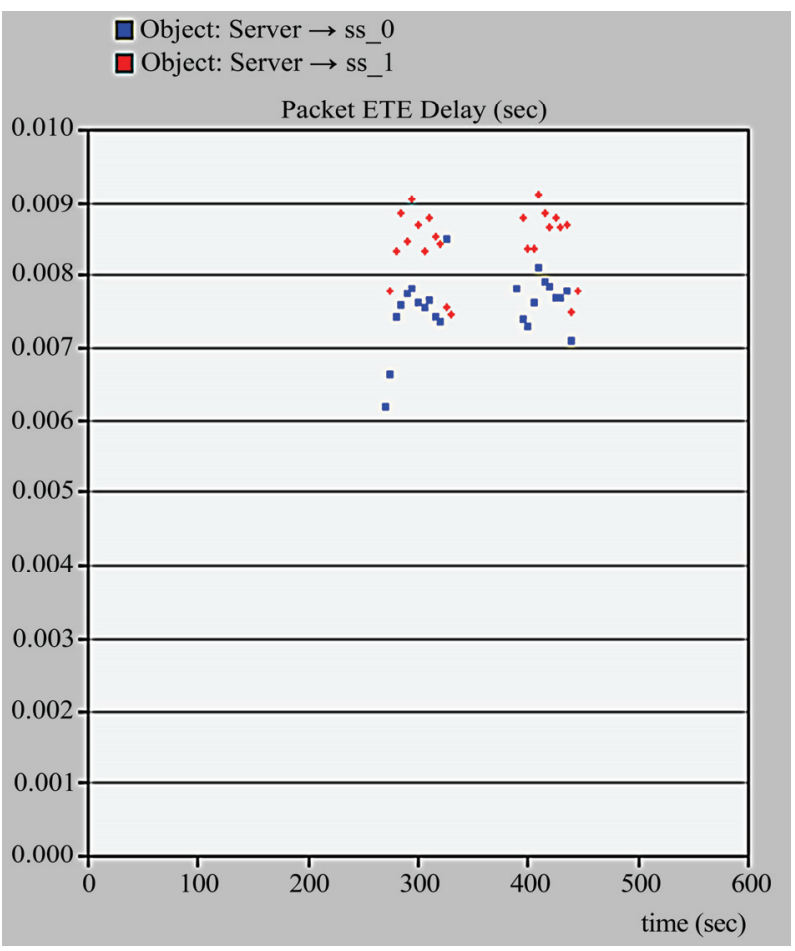

Figure 10. End -to -end packet delay (adaptive).

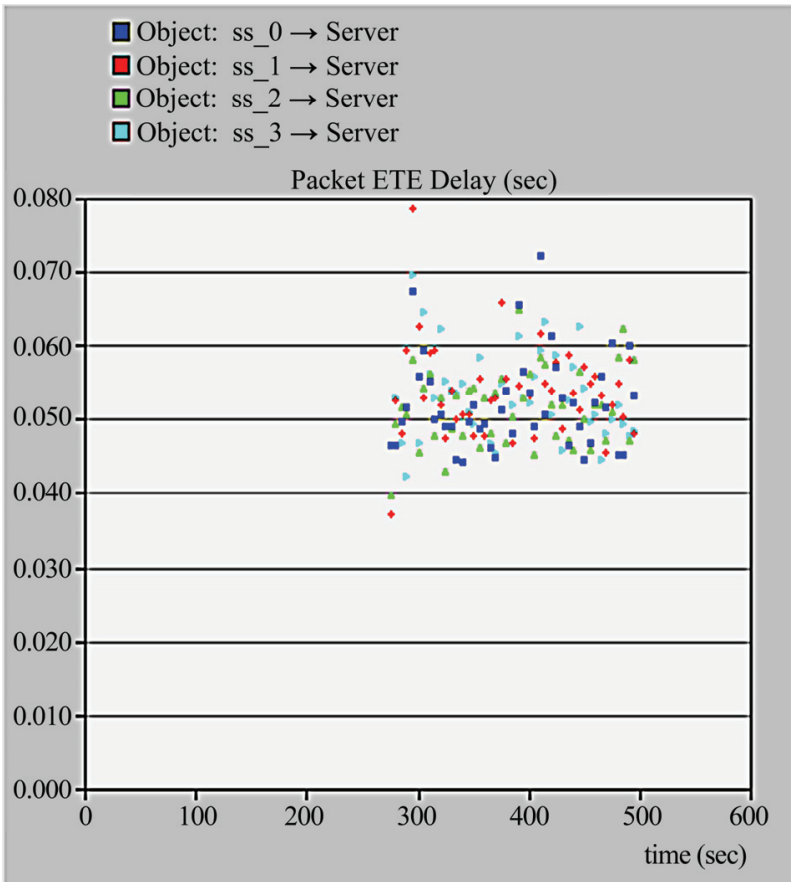

Figure 12. End-to-end packet delay (UL).

\section{Conclusions}

This paper presents a dynamic strategy that takes advantage of underutilized DL sub-frame, allowing the UL sub-frame to acquire temporarily free resources of DL 


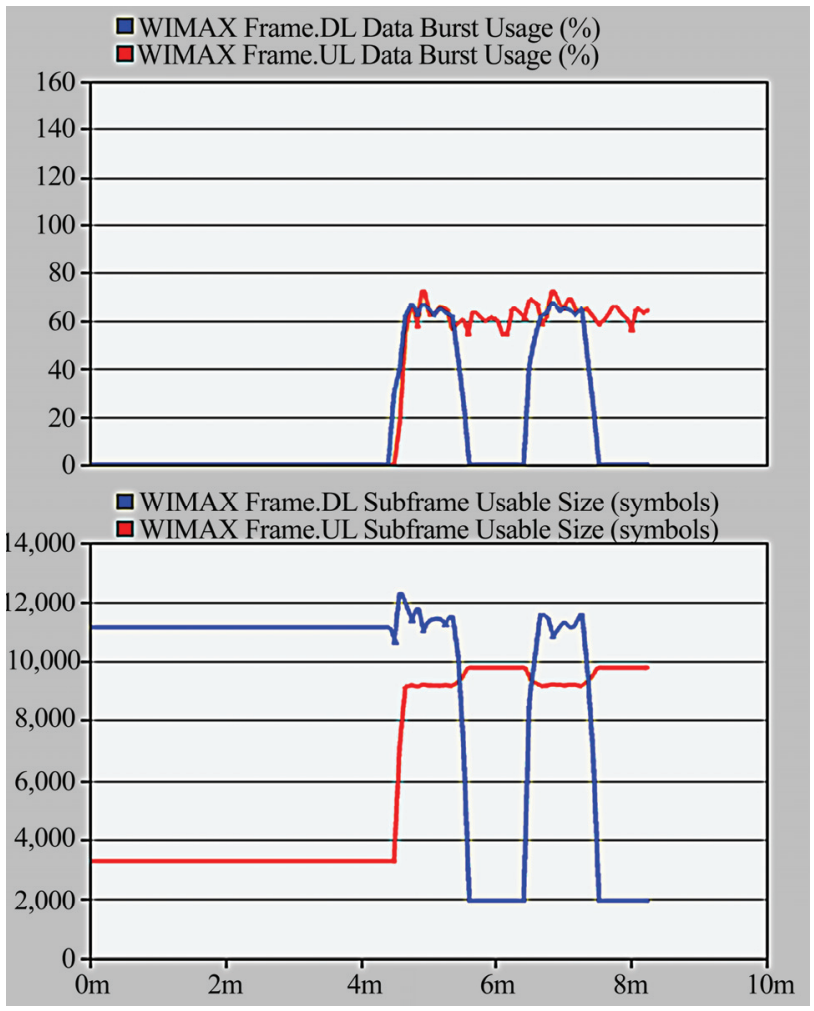

Figure 13. Data burst usage and sub-frame usable size.

when UL observes shortage of resources. Our proposed mechanism ensures the performance of DL to remain unaffected by continuous monitoring of the DL utilization. In case of requirements, the sub-frame boundaries could be readjusted and DL will be prioritized. The proposed mechanism is tested under controlled environment using OPNET Modeler for correctness and effectiveness.

In our proposed work, an observable improvement is seen in both throughput and end-to-end packet delay at $\mathrm{UL}$ without affecting the performance of DL.

\section{References}

[1] C. Eklund, et al., "IEEE standard 802.16: A technical overview of the wireless MAN air interface for broadband wireless access," IEEE Communication Magazines, pp. 98-107, June 2002.

[2] A. Ghosh, et al., "Broadband wireless access with WiMAX/802.16: Current performance benchmarks and future potential," IEEE Communication Magazine, pp. 129-36, February 2005.

[3] 802.16d Task Group, "IEEE standard for local and metropolitan area networks part 16: Air interface for fixed broadband wireless access systems," IEEE, IEEE80216-2004 Version, 802.16d or Fixed WMAN, June 2004.

[4] 802.16e Task Group, "IEEE standard for local and metropolitan area networks part 16: Air interface for mobile broadband wireless access systems," IEEE, Active International Standard, 802.11e or Mobile WMAX, 2005.

[5] WiMAX Forum: Mobile WiMAX - Part I: A Technical Overview and Performance Evaluation, June 2006.

[6] X. Yang, M. Venkatachalam, and S. Mohanty, "Exploiting the MAC layer flexibility of WiMAX to systematically enhance TCP performance," IEEE Mobile WiMAX Symposium, 2007.

[7] K. C. Chen and J. Roberto B. de Marca, "Mobile WiMAX," IEEE PRESS, IEEE Communications Society, Sponsor, John Wiley \& Sons, Ltd, April 2008.

[8] J. Sun, Y. Yao, and H. Zhu, "Quality of service scheduling for 802.16d broadband wireless access systems," Proceedings of IEEE 63rd International Conference of Vehicular Technology 2006, VTC 2006-Spring, Vol. 3, pp. 1221-1225, 2006.

[9] G. Chu, D. Wang, and S. Mei, "A QoS architecture for the MAC protocol of IEEE 802.16d BWA system," Proceedings of IEEE International Conference on Communications, Circuits and Systems, Vol. 1, pp. 435-439, June 2002.

[10] K. Wongthavarawatn and A. Ganz, "Packet scheduling for QoS support in IEEE 802.16d broadband wireless access systems," International Journal of Communication Systems, Vol. 16, pp. 81-96, 2003.

[11] J. Chen, W. Jiao, and H. Wang, "A service flow management strategy for IEEE $802.16 \mathrm{~d}$ broadband wireless access systems in TDD mode," Proceedings of IEEE International Conference on Communications 2005, Vol. 5 , pp. 3422-3426, May 2005.

[12] R. Iyengar, P. Iyer, and B. Sikdar. "Delay analysis of 802.16 based last mile wireless networks," IEEE Globecom, USA, 2005.

[13] R. Pries, D. Staehle, and D. Marsico, "IEEE 802.16 capacity enhancement using an adaptive TDD split," Vehicular Technology Conference, pp. 1539-1543, May 11-14, 2008.

[14] A. Q. Ansari, A. Q. K. Rajput, and M. Hashmani; "WiMAX network optimization-analyzing effects of adaptive modulation and coding schemes used in conjunction with ARQ and HARQ," Proceedings of 7th International Conference on Communication Networks and Services Research, pp. 6-13, 2009.

[15] M. Ma, J. Lu, S. Kumar, and B. Chong, "A three-tier framework and scheduling to support QoS service in WiMAX," Proceedings of ICICS, 2007.

[16] "Understanding WiMAX model internals and interfaces," Opnetwork, 2008. 